\title{
A Review on: Opinion Mining and Sentiment Analysis based on Natural Language Processing
}

\author{
Swati N. Manke \\ JSPM'S BSIOTR \\ Wagholi, \\ Savitribai Phule Pune University
}

\author{
Nitin Shivale \\ JSPM'S BSIOTR \\ Wagholi, \\ Savitribai Phule Pune University
}

\begin{abstract}
In marketing and advertising domains Opinion Mining is being larger domain. Advertiser needs to analyze performance/ popularity of ads that he/she posted on site. Star rating based mechanism may go fraud, because of robots or automatic responders. So, current system needs to be analyzed using comments \& natural language processing. Fraud comments could be removed by using irrelevant comment removal mechanism suggested in paper. In this paper the role and importance of social networks as preferred environments for opinion mining and sentiment analysis are discussed especially. In this paper, selected properties of social networks that are relevant with respect to opinion mining are briefly described and outline the general relationships between the two disciplines. It presents the related work and provide basic definitions used in opinion mining area. Then, the original method of opinion classification is introduce and test the presented algorithm on real world datasets acquired from popular Polish social networks, reporting on the results. The results are outperform and soundly support the main issue of the paper, that social networks exhibit properties that make them very suitable for opinion mining activities.
\end{abstract}

\section{Keywords}

Opinion, Sentiment, Domain-dependent corpus, Domainindependent corpus, Relevance.

\section{INTRODUCTION}

In the past few years due to many challenging research problems and practical applications opinion mining (or sentiment analysis) has attracted a great deal of attention from researchers of natural language processing and data mining. Opinion lexicon expansion and opinion target extraction are two fundamental problems in opinion mining. An opinion lexicon is a list of opinion words such as good, excellent, poor, and bad used to indicate positive or negative sentiments. Sentence and document sentiment classification and featurebased opinion summarization forms the foundation of many opinion mining tasks. Opinion targets are related to the topics on which opinions are expressed. They are important because without knowing the targets, the opinions expressed in a sentence or document are of limited use. The computational treatment of opinion, sentiment, and subjectivity has recently attracted a great deal of attention, in part because of its potential applications. It also has proven useful for companies, recommender systems, and editorial sites to create summaries of people's experiences and opinions that consist of subjective expressions extracted from reviews or even just a review's polarity positive or negative. Data-driven methods, resisting traditional text-categorization techniques are document polarity classification poses a significant challenge.

Opinion Mining is being larger domain of the marketing and advertising domains. Advertiser needs to analyze performance/ popularity of ads that he/she posted on site. Star rating based mechanism may go fraud, because of robots or automatic responders. So, current system needs to be analyzed using comments \& natural language processing. Fraud comments could be removed by using irrelevant comment removal mechanism suggested in paper.

In this paper the role and importance of social networks as preferred environments for opinion mining and sentiment analysis especially. Briefly, describing selected properties of social networks that are relevant with respect to opinion mining and outline the general relationships between the two disciplines. In this paper the related work and provide basic definitions used in opinion mining is presented. Then, introduce the original method of opinion classification and test the presented algorithm on real world datasets acquired from popular Polish social networks, reporting on the results. The results are promising and soundly support the main thesis of the paper, namely, that social networks exhibit properties that make them very suitable for opinion mining activities.

\section{RELATED WORK}

The document, sentence, or even phrase (word) levels can be generally analyzed Opinions and sentiments expressed in text reviews. The document-level (sentence-level) opinion mining is used to classify the overall subjectivity or sentiment expressed in an individual review document (sentence).

Wei Jin and Hung Hay Ho [2] proposed a novel and robust machine learning approach for web opinion mining and extraction. This model provides solutions for server problems that have been not provided by previous approaches. This system can self-learn new vocabularies based on the pattern its has learned, which is used in text and web mining. A novel bootstrapping approach is used to handle situations in which colleting a large training set could be expensive and difficult to accomplices. In this paper the effectiveness of proposed approach in web opinion mining and extraction from product review are demonstrated in result.

Guang Qiu, Bing Liu, Jiajun Bu and Chun Chen [4] focuses on to important tasks in opinion mining that are opinion lexicon expansion and target extraction. In this paper, a propagation approach to extract opinion words and targets iteratively given only a seed opinion lexicon of small size. The identified relations between opinion words and targets are used for extraction in this paper a novel method for new opinion words polarity assignment and noisy targe priming are proposed. The new approach is compared with others on standard testing data set. The result of this paper shows that this approach out performance other state-of-the-art methods.

Bo Pang and Lillian Lee [6] examine the relation between subjectivity detection and polarity classification. The subjectivity detection can compress reviews in shorter extracts 
that still retains polarity information at a level comparable to that of the full review. By using Naive Bayes polarity classifier the subjectivity extract are shown to be more effective input than the originating document. The paper shows that the minimum-cut framework results in the development of efficient algorithm for sentiment analysis. Via this framework contextual information can lead to statistically significant improvement in polarity classification accuracy.

Niklas Jacob and Iryna Gurevych [3] show how a CRF-based approach for opinion target extraction in a single and cross domain setting. In this paper a comparative evaluation of this approach on data set from four different domains are presented. The CRF-based approach out performance a supervised baseline on all dataset in the single domain setting. The CRF-based approach also yields promising results in the cross domain setting.

Maniquing $\mathrm{Hu}$ and Bing Liu [7] proposed a number of techniques for mining features from product reviews based on data mining and natural language processing method. To produce feature based summary of a large number of customer review of a product sold online is the objective of this paper. Opinion mining becomes increasingly important as more people are buying and expressing their opinion on the web. Experimental result of this paper indicate that the propose techniques are effective in performing tasks.

Opinion mining based on document, sentence, or phrase (word) level does not represent what exactly people like or dislike.

\subsection{Extraction of Opinion Features}

A subproblem of opinion mining is opinion feature extraction, with the vast majority of existing work. Previous approaches of opinion mining are classified into two supervised and unsupervised.

Supervised learning models including hidden Markov models and conditional random fields have been used to tag features or aspects of commented entities. Supervised models can be carefully tuned to perform well on a particular domain, but need extensive retraining when applied to a different domain. A decent-sized set of labeled data is generally needed for model learning on every domain.

In review sentences, an unsupervised NLP approaches extract opinion features by mining syntactic patterns of features are implied. The approaches attempt to discover syntactic relations among feature terms and opinion words in sentences by using carefully crafted syntactic rules, or semantic role labeling. Syntactic relations identified by the methods helps to locate features associated with opinion words, but could also inadvertently extract large number of invalid features due to the colloquial nature of online reviews.

The results of statistical analysis on a given corpus to understand the distributional characteristics of opinion features, unsupervised corpus statistics approaches used. This approaches resistance to the colloquial nature of online reviews given a suitably large review corpus. $\mathrm{Hu}$ and Liu proposed [ 8] an association rule mining (ARM) approach to mine frequent item sets as potential opinion features, which are nouns and noun phrases with high sentence-level frequency (or support). ARM, relies on the frequency of item sets, has the some limitations for the task of feature identification, 1) frequent but invalid features are extracted incorrectly, and 2) rare but valid features may be overlooked.
To address feature-based opinion mining problems, a mutual reinforcement clustering (MRC) introduced by $\mathrm{Su}$ et al. [13] this approach is used to mine the associations between feature categories and opinion word groups, based on a co occurrence weight matrix generated from the given review corpus. MRC is able to extract infrequent features, provided that the mutual relationships between feature and opinion groups found during the clustering phase is accurate which is unlike to other methods. Due to the difficulty in obtaining good clusters on real-life reviews, MRC's precision is low.

The existing approaches to feature extraction only use the knowledge or patterns mined from a given single review corpus, by completely ignoring the possible variations present in a different domain-independent corpus. In this paper, IEDR approach utilizes the fact that word distribution characteristics vary across different types of corpora, in particular domainspecific versus domain-independent, to derive powerful hints that help discriminate valid features from the invalid ones. In the first step of this approach, some syntactic dependence rules to extract candidate features, similar to NLP approaches are defined. In the second step, employ the IEDR measures to identify the desired domain-specific opinion features. The key difference between IEDR compared to existing methods lies in its smart fusion of domain-dependent and domainindependent information sources.

\section{METHODOLOGIES PROPOSED FOR OPINION MINING}

Opinion features such as reviews on a particular product are typically domain-specific. The feature appears frequently in the given review domain, and which are outside the domain is domain-independent corpus about product. Domain-specific opinion features are mentioned more frequently in the domain corpus of reviews, as compared to a domain-independent corpus. A domain-dependent review corpus and a domainindependent corpus is observed. Figure 1 shows that, first extract a list of candidate features from the review corpus by defining manually syntactic rules. Each extracted candidate feature, will estimate its IDR, which represents the statistical association of the candidate to the given domain corpus, and extrinsic-domain relevance, will reflects the statistical relevance of the candidate to the domain-independent corpus. Only candidates with IDR scores more exceeding a predefined intrinsic relevance threshold and EDR scores less than another extrinsic relevance threshold are extracted as valid opinion features. In short, this paper identifies opinion features that are domain-specific and at the same time domain-independent corpus are removed and ignored.

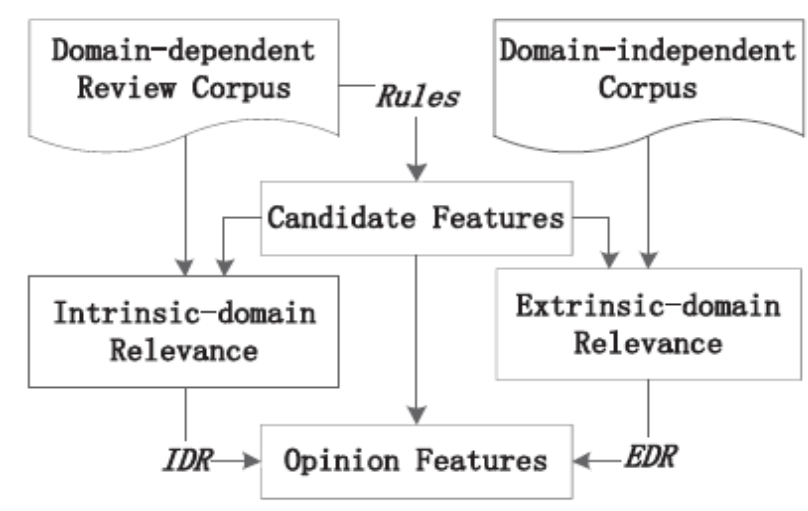

Fig. 1: IEDR Workflow 


\subsection{Candidate Feature Extraction}

Opinion features appear as the subject or object of a review sentence are generally nouns or noun phrases. In the dependence grammar, the subject opinion feature has a syntactic relationship of type subject verb with the sentence predicate. The object opinion feature has a dependence relationship of verb-object on the predicate. It also has a dependence relationship of preposition-object on the prepositional word in the sentence.

\subsection{Opinion Feature Extraction}

Domain relevance characterizes how much a term is related to a particular corpus based on two kinds of statistics, dispersion and deviation. Dispersion identifies how significantly a term is mentioned in overall documents by measuring the distributional significance of the term across different documents in the entire domain. Deviation results about how frequently a term is mentioned in a particular document by measuring its distributional significance in the document. Both dispersion and deviation are calculated using the frequency-inverse document frequency term weights which is a well known technique.

\section{PROPOSED SYSTEM ARCHITECTURE}

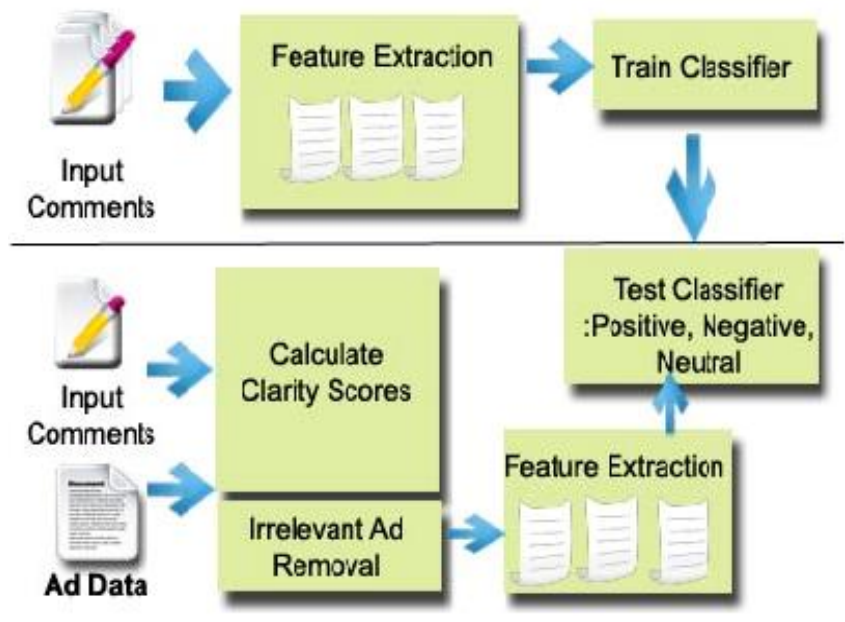

Fig 2. System Architecture

The system Architecture of proposed system is as shown in the following figure. In first part of the system, it is shown that input will be collected from various online shopping websites such as amazon, flipcart, snapdeal, Jabong etc. The comments which are written by the customers about any product in textural format in natural language is collected and those comments are used for feature extraction. After feature extraction the comments are passed to the trainer classifier for finding the patterns of comments. To identify patterns, techniques like N-Gram Extraction and part of speech Extraction are used by the trainer classifier. Collection comments and identifying patterns of comments is the online process of this system.

In second part of figure of the system offline process is shown. From the collected comments which are in natural language textural format the irrelevant comments are removed and clarity score is calculated. To remove irrelevant comments KL Divergence algorithm is used and clarity score in also calculated using a threshold value. In this process the domaindependent features and domain-independent comments are separated for feature extraction. In feature extraction NER tagger, Naive Bays classifier and porter streaming algorithms are used.

With the help of trainer classifier and feature extraction the test classifier gives the feedback about a specified product as positive, negative and neutral.

\section{CONCLUSION}

In Future by implementing this paper the social networks can give perfect solution to the problem of opinion acquisition and dissemination and perceived as natural enablers for opinion mining applications. In this paper, concept presented a proof of, examples of analysis that aim at gathering user opinions in two different application areas. Both experiments suggest that the networks fuelling the websites in question provide relevant context for opinion mining. The system aware of the fact that has not utilized the information from the social network directly in the opinion mining algorithm. Merely, the system has tested the ability to attain high accuracy and quality of sentiment prediction using the data harvested from a social network site. It includes the user's reception of opinions contained in the text and further improvements of the presented all expect to attain the improvement of classification performance due to the utilization of information derived from the social networks, namely, the information on relationships and connections between users. We also intend to develop an active learning strategy for this type of classification task. The system suggested by this paper can be used in online marketing field as well as advertising field. The same system can be also used in any field where feedback about service can be collected. For example in hotels, railway services, about teacher. It can be also implemented for different languages

\section{ACKNOWLEDGMENTS}

To prepare this survey paper, I would like to be very thankful to my project guide Prof. Nitin Shivale, our M.E. Coordinator Prof. Archana Lomte And Head of the Department Prof.G.M.Bhandari in Computer Department of Bhivarabai Savant Institute of Technology \& Research, Wagholi, Affiliated to Savitribai Phule University.

I would also like to thank the whole IEEE organization who helps allot to search various research papers related to my research. Because of their support only I am able to complete my research note.

\section{REFERENCES}

[1] Zhen Hai, Kuivyu , Jung-Jae Kim, and Christopher C. Yang "Identify Features in opinion mining via intrinsic and extrinsic Domain Relevance" Vol 26, no.3, March 2014.

[2] W. Jin and H.H. Ho, "A Novel Lexicalized HMM-Based Learning Framework for Web Opinion Mining," Proc. 26th Ann. Int'l Conf. Machine Learning, pp. 465-472, 2009.

[3] N. Jakob and I. Gurevych, "Extracting Opinion Targets in a Single and Cross-Domain Setting with Conditional Random Fields," Proc. Conf. Empirical Methods in Natural Language Processing, pp. 1035-1045, 2010.

[4] G. Qiu, B. Liu, J. Bu, and C. Chen, "Opinion Word Expansion and Target Extraction through Double Propagation," Computational Linguistics, vol. 37, pp. 9 27, 2011. 
[5] Sannella, M. J. . M. Kim and E. Hovy, "Extracting Opinions Holders, and Topics Expressed in Online News Media Text," Proc. ACL/COLING Workshop Sentiment and Subjectivity in Text, 2006.

[6] Forman, B. Pang and L. Lee, "A Sentimental Education: Sentiment Analysis Using Subjectivity Summarization Based on Minimum Cuts," Proc. 42nd Ann. Meeting on Assoc. for Computational Linguistics.

[7] Brown, L. D., Hua, M. Hu and B. Liu, "Mining and Summarizing Customer Reviews," Proc. 10th ACM SIGKDD Int'l Conf. Knowledge Discovery and Data Mining, pp. 168-177, 2004.

[8] B. Liu, "Sentiment Analysis and Opinion Mining," Synthesis Lectures on Human Language Technologies, vol. 5, no. 1, pp. 1-167,May 2012

[9] Y. Jo and A.H. Oh, "Aspect and Sentiment Unification Model for Online Review Analysis," Proc. Fourth ACM Int'l Conf. Web Search and Data Mining, pp. 815-824, 2011.
[10] G. Qiu, C. Wang, J. Bu, K. Liu, and C. Chen, "Incorporate the Syntactic Knowledge in Opinion Mining in User-Generated Content," Proc. WWW 2008 Workshop NLP Challenges in the Information Explosion Era, 2008

[11] A. Popescu and O. Etzioni, "Extracting Product Features and Opinions from Reviews," Proc. Human Language Technology Conf. and Conf. Empirical Methods in Natural Language Processing, pp. 339- 346, 2005.

[12] V. Hatzivassiloglou and J.M. Wiebe, "Effects of Adjective Orientation and Gradability on Sentence Subjectivity," Proc. $18^{\text {th }}$ Conf. Computational Linguistics, pp. 299-305, 2000.

[13] Q. Su, X. Xu, H. Guo, Z. Guo, X. Wu, X. Zhang, B. Swen, and Z. Su,"Hidden Sentiment Association in Chinese Web Opinion Mining,"Proc. 17th Int'l Conf. World Wide Web, pp. 959-968, 2008 